Acta Crystallographica Section E

Structure Reports

Online

ISSN 1600-5368

\section{N-(4-Methylphenylsulfonyl)-3-nitro- benzamide}

\section{S. Sreenivasa, ${ }^{\text {a }}$ M. S. Nanjundaswamy, ${ }^{\text {b }}$ A. G. Sudha,} K. J. Pampa, ${ }^{d}$ N. K. Lokanath ${ }^{\mathrm{e}}$ and P. A. Suchetan ${ }^{\mathrm{f}_{*}}$

a Department of Studies and Research in Chemistry, Tumkur University, Tumkur, Karnataka 572 103, India, 'bepartment of Chemistry, AVK College for Women, Davangere-2, India, ${ }^{\mathbf{C}}$ University College of Science, Tumkur University, Tumkur, India, ${ }^{\mathbf{d}}$ Department of Studies in Microbiology, University of Mysore,

Manasagangotri, Mysore, India, ${ }^{\mathbf{e}}$ Department of Studies in Physics, University of Mysore, Manasagangotri, Mysore, India, and 'Department of Studies and Research in Chemistry, U.C.S., Tumkur University, Tumkur, Karnataka 572 103, India Correspondence e-mail: pasuchetan@yahoo.co.in

Received 15 January 2014; accepted 19 January 2014

Key indicators: single-crystal X-ray study; $T=293 \mathrm{~K}$; mean $\sigma(\mathrm{C}-\mathrm{C})=0.003 \AA$; $R$ factor $=0.039 ; w R$ factor $=0.114 ;$ data-to-parameter ratio $=11.7$.

In the title compound, $\mathrm{C}_{14} \mathrm{H}_{12} \mathrm{~N}_{2} \mathrm{O}_{5} \mathrm{~S}$, the dihedral angle between the aromatic rings is $86.29(1)^{\circ}$ and the conformation between the $\mathrm{C}=\mathrm{O}$ bond of the amide group and the meta $-\mathrm{NO}_{2}$ group is syn. The $\mathrm{C}-\mathrm{S}-\mathrm{N}-\mathrm{C}$ torsion angle is $-65.87(19)^{\circ}$ and the molecule has an L-shaped conformation. In the crystal, the molecules are connected into inversion dimers through pairs of $\mathrm{N}-\mathrm{H} \cdots \mathrm{O}$ hydrogen bonds and $\mathrm{C}-\mathrm{H} \cdots \mathrm{O}$ interactions forming $R_{2}^{2}(8)$ and $R_{2}^{2}(14)$ loops, respectively. The dimers are connected by further $\mathrm{C}-\mathrm{H} \cdots \mathrm{O}$ interactions, thereby forming (100) sheets.

\section{Related literature}

For related structures see: Suchetan et al. (2010, 2011, 2012).

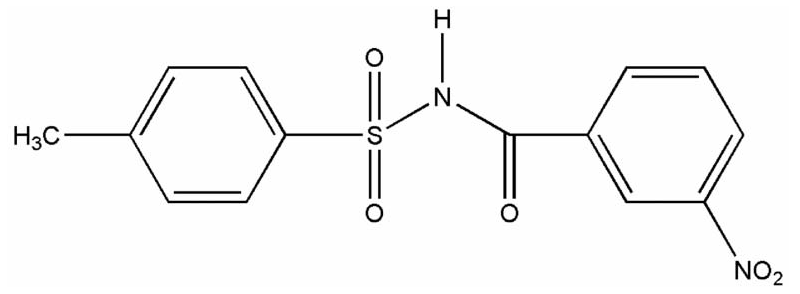

\section{Experimental}

Crystal data

$\mathrm{C}_{14} \mathrm{H}_{12} \mathrm{~N}_{2} \mathrm{O}_{5} \mathrm{~S} \quad M_{r}=320.32$
Monoclinic, $P 2_{1} / c$

$a=4.9736(5) \AA$

$b=23.245$ (2) $\AA$

$c=12.7197$ (11)

$\beta=100.820(4)^{\circ}$

$V=1444.4(2) \AA^{3}$

\section{Data collection}

Bruker APEXII diffractometer Absorption correction: multi-scan (SADABS; Bruker, 2009)

$T_{\min }=0.481, T_{\max }=0.638$

$Z=4$

$\mathrm{Cu} K \alpha$ radiation

$\mu=2.24 \mathrm{~mm}^{-1}$

$T=293 \mathrm{~K}$

$0.39 \times 0.29 \times 0.20 \mathrm{~mm}$

Refinement

$R\left[F^{2}>2 \sigma\left(F^{2}\right)\right]=0.039$

$w R\left(F^{2}\right)=0.114$

$S=1.06$

2378 reflections

204 parameters

12115 measured reflections 2378 independent reflections 2053 reflections with $I>2 \sigma(I)$ $R_{\text {int }}=0.035$

Table 1

Hydrogen-bond geometry $\left(\AA{ }^{\circ}\right)$.

\begin{tabular}{lllll}
\hline$D-\mathrm{H} \cdots A$ & $D-\mathrm{H}$ & $\mathrm{H} \cdots A$ & $D \cdots A$ & $D-\mathrm{H} \cdots A$ \\
\hline $\mathrm{N} 1-\mathrm{H} N 1 \cdots \mathrm{O} 2^{\mathrm{i}}$ & $0.80(3)$ & $2.14(3)$ & $2.927(3)$ & 167 \\
$\mathrm{C} 13-\mathrm{H} 13 \cdots \mathrm{O} 2^{\mathrm{i}}$ & 0.93 & 2.59 & $3.333(3)$ & 137 \\
$\mathrm{C} 3-\mathrm{H} 3 \cdots \mathrm{O} 4{ }^{\mathrm{ii}}$ & 0.93 & 2.58 & $3.459(3)$ & 155 \\
\hline
\end{tabular}

Symmetry codes: (i) $-x+2,-y+1,-z+1$; (ii) $-x, y-\frac{1}{2},-z+\frac{1}{2}$.

Data collection: APEX2 (Bruker, 2009); cell refinement: APEX2 and SAINT-Plus (Bruker, 2009); data reduction: SAINT-Plus and XPREP (Bruker, 2009); program(s) used to solve structure: SHELXS97 (Sheldrick, 2008); program(s) used to refine structure: SHELXL97 (Sheldrick, 2008); molecular graphics: Mercury (Macrae et al., 2008); software used to prepare material for publication: SHELXL97.

The authors acknowledge the IOE X-ray diffractometer facility, University of Mysore, Mysore, for the data collection.

Supporting information for this paper is available from the IUCr electronic archives (Reference: HB7184).

\section{References}

Bruker (2009). APEX2, SADABS, SAINT-Plus and XPREP. Bruker AXS Inc., Madison, Wisconsin, USA.

Macrae, C. F., Bruno, I. J., Chisholm, J. A., Edgington, P. R., McCabe, P., Pidcock, E., Rodriguez-Monge, L., Taylor, R., van de Streek, J. \& Wood, P. A. (2008). J. Appl. Cryst. 41, 466-470.

Sheldrick, G. M. (2008). Acta Cryst. A64, 112-122.

Suchetan, P. A., Foro, S. \& Gowda, B. T. (2011). Acta Cryst. E67, o917.

Suchetan, P. A., Foro, S. \& Gowda, B. T. (2012). Acta Cryst. E68, o1507.

Suchetan, P. A., Gowda, B. T., Foro, S. \& Fuess, H. (2010). Acta Cryst. E66, o1039. 


\section{supporting information}

Acta Cryst. (2014). E70, o191 [doi:10.1107/S1600536814001317]

\section{N-(4-Methylphenylsulfonyl)-3-nitrobenzamide}

\section{S. Sreenivasa, M. S. Nanjundaswamy, A. G. Sudha, K. J. Pampa, N. K. Lokanath and P. A. Suchetan}

\section{S1. Introduction}

As a part of our continued efforts to study the crystal structures of N-(aroyl)-arylsulfonamides (Suchetan et al., 2010, 2011, 2012), we report here the crystal structure of the title compound (I) (Fig 1).

\section{S2. Experimental}

\section{S2.1. Synthesis and crystallization}

The title compound (I) was prepared by refluxing a mixture of 3-nitrobenzoic acid, 4-methylbenzenesulfonamide and phosphorous oxychloride $\left(\mathrm{POCl}_{3}\right)$ for $2 \mathrm{~h}$ on a water bath. The resultant mixture was cooled and poured into ice cold water. The solid obtained was filtered and washed thoroughly with water and then dissolved in sodium bicarbonate solution. The compound was later reprecipitated by acidifying the filtered solution with dilute $\mathrm{HCl}$. The compound obtained was filtered and later dried (Melting point: $459 \mathrm{~K}$ ).

Colorless prisms of (I) were obtained from a slow evaporation of its aqueous methanolic solution at room temperature.

\section{S2.2. Refinement}

The $\mathrm{H}$ atom of the $\mathrm{NH}$ group was located in a difference map and later refined freely. The other $\mathrm{H}$ atoms were positioned with idealized geometry using a riding model with $\mathrm{C}-\mathrm{H}=0.93-0.96 \AA$. All $\mathrm{H}$ atoms were refined with isotropic displacement parameters (set to 1.2-1.5 times of the Ueq of the parent atom).

\section{S3. Results and discussion}

In I, the dihedral angle between the two aromatic rings is $86.29(1)^{\circ}$. Compared to this, the dihedral angle is $79.4(1)^{\circ}$ in $\mathrm{N}$-(4-methylphenylsulfonyl)-benzamide (II) (Suchetan et al., 2010), 89.8 (1) ${ }^{\circ}$ in N-(4-methylphenylsulfonyl)-4-nitrobenzamide (III) (Suchetan et al., 2011) and 86.9 (2) in N-(phenylsulfonyl)-3-nitrobenzamide (IV) (Suchetan et al., 2012). Thus, introducing a nitro group into the benzoyl ring results in an increase of the dihedral angle between the aromatic rings. The conformation between the $\mathrm{N}-\mathrm{H}$ bond and the meta- $\mathrm{NO}_{2}$ group is anti in contrast to the syn conformation observed in IV (Suchetan et al., 2012). The molecule is twisted at the S atom, the dihedral angle between the planes defined by the $\mathrm{S}-\mathrm{N}-\mathrm{C}=\mathrm{O}$ segment in the central chain and the sulfonyl benzene rings being $79.16(1)^{\circ}$.

In the crystal structure, the molecules are connected into inversion dimers through $\mathrm{N} 1-\mathrm{HN} 1 \cdots \mathrm{O} 2$ hydrogen bonds (Table 1, Figure 2) and $\mathrm{C} 13-\mathrm{H} 13 \cdots \mathrm{O} 2$ interactions forming $\mathrm{R}_{2}{ }^{2}(8)$ and $\mathrm{R}_{2}{ }^{2}(14)$ ring motifs respectively. These dimers are further connected into $\mathrm{C}(12)$ chains through $\mathrm{C} 3-\mathrm{H} 3 \cdots \mathrm{O} 4$ interactions forming sheets (Figure 2). 


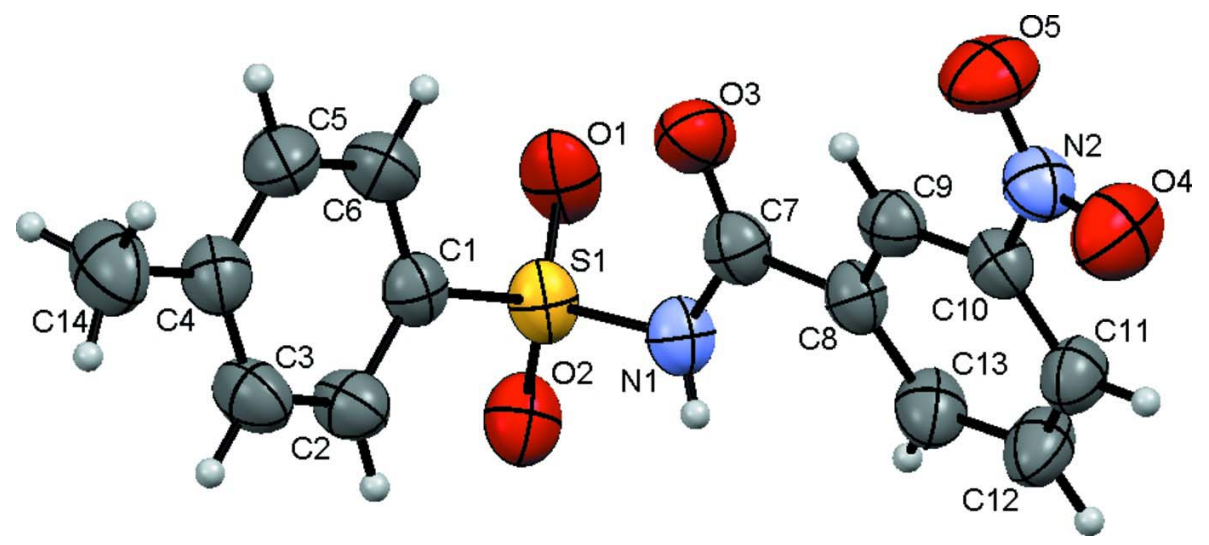

Figure 1

Molecular structure of the title compound, showing displacement ellipsoids at the $50 \%$ probability level.

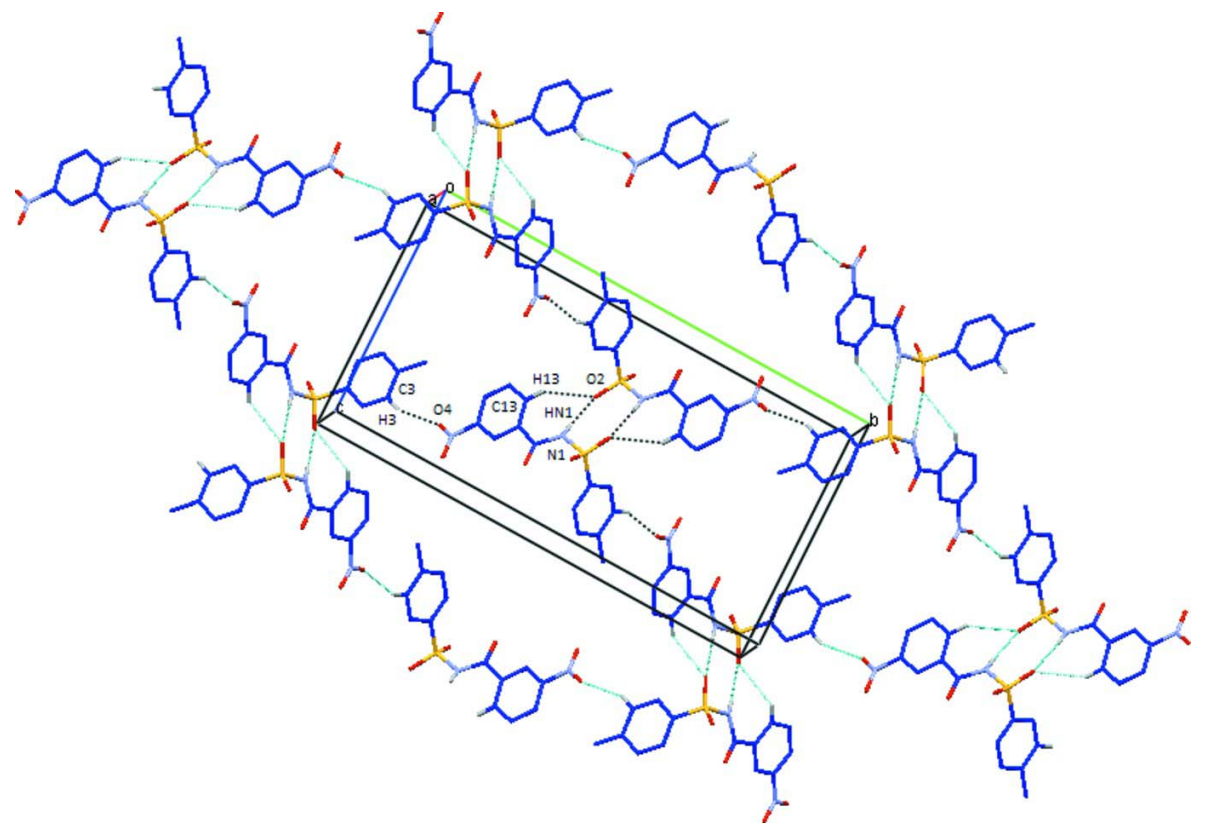

Figure 2

Formation of sheets in the crystal structure.

N-(4-Methylphenylsulfonyl)-3-nitrobenzamide

Crystal data

$\mathrm{C}_{14} \mathrm{H}_{12} \mathrm{~N}_{2} \mathrm{O}_{5} \mathrm{~S}$

$M_{r}=320.32$

Monoclinic, $P 2_{1} / c$

Hall symbol: -P 2ybc

$a=4.9736(5) \AA$

$b=23.245(2) \AA$

$c=12.7197(11) \AA$

$\beta=100.820(4)^{\circ}$

$V=1444.4(2) \AA^{3}$

$Z=4$

$F(000)=664$
Prism

$D_{\mathrm{x}}=1.473 \mathrm{Mg} \mathrm{m}^{-3}$

Melting point: $459 \mathrm{~K}$

$\mathrm{Cu} K \alpha$ radiation, $\lambda=1.54178 \AA$

Cell parameters from 1127 reflections

$\theta=3.8-64.5^{\circ}$

$\mu=2.24 \mathrm{~mm}^{-1}$

$T=293 \mathrm{~K}$

Prism, colourless

$0.39 \times 0.29 \times 0.20 \mathrm{~mm}$ 


\section{Data collection}

Bruker APEXII

diffractometer

Radiation source: fine-focus sealed tube

Graphite monochromator

phi and $\varphi$ scans

Absorption correction: multi-scan

(SADABS; Bruker, 2009)

$T_{\text {min }}=0.481, T_{\max }=0.638$

12115 measured reflections

\section{Refinement}

Refinement on $F^{2}$

Least-squares matrix: full

$R\left[F^{2}>2 \sigma\left(F^{2}\right)\right]=0.039$

$w R\left(F^{2}\right)=0.114$

$S=1.06$

2378 reflections

204 parameters

0 restraints

Primary atom site location: structure-invariant

direct methods
2378 independent reflections

2053 reflections with $I>2 \sigma(I)$

$R_{\text {int }}=0.035$

$\theta_{\max }=64.5^{\circ}, \theta_{\min }=3.8^{\circ}$

$h=-5 \rightarrow 4$

$k=-26 \rightarrow 26$

$l=-14 \rightarrow 14$

2 standard reflections every 1 reflections

intensity decay: $1 \%$

Secondary atom site location: difference Fourier map

Hydrogen site location: inferred from

neighbouring sites

$\mathrm{H}$ atoms treated by a mixture of independent and constrained refinement

$w=1 /\left[\sigma^{2}\left(F_{\mathrm{o}}^{2}\right)+(0.0621 P)^{2}+0.3691 P\right]$

where $P=\left(F_{\mathrm{o}}^{2}+2 F_{\mathrm{c}}^{2}\right) / 3$

$(\Delta / \sigma)_{\max }<0.001$

$\Delta \rho_{\max }=0.20 \mathrm{e}^{-3}$

$\Delta \rho_{\min }=-0.23$ e $\AA^{-3}$

\section{Special details}

Geometry. All s.u.'s (except the s.u. in the dihedral angle between two l.s. planes) are estimated using the full covariance matrix. The cell s.u.'s are taken into account individually in the estimation of s.u.'s in distances, angles and torsion angles; correlations between s.u.'s in cell parameters are only used when they are defined by crystal symmetry. An approximate (isotropic) treatment of cell s.u.'s is used for estimating s.u.'s involving l.s. planes.

Refinement. Refinement of $F^{2}$ against ALL reflections. The weighted $R$-factor $w R$ and goodness of fit $S$ are based on $F^{2}$, conventional $R$-factors $R$ are based on $F$, with $F$ set to zero for negative $F^{2}$. The threshold expression of $F^{2}>2 \sigma\left(F^{2}\right)$ is used only for calculating $R$-factors (gt) etc. and is not relevant to the choice of reflections for refinement. $R$-factors based on $F^{2}$ are statistically about twice as large as those based on $F$, and $R$ - factors based on ALL data will be even larger.

Fractional atomic coordinates and isotropic or equivalent isotropic displacement parameters $\left(\AA^{2}\right)$

\begin{tabular}{lllll}
\hline & $x$ & $y$ & $z$ & $U_{\text {iso }} * / U_{\text {eq }}$ \\
\hline S1 & $1.10155(10)$ & $0.50190(2)$ & $0.32699(4)$ & $0.0546(2)$ \\
C8 & $0.6073(4)$ & $0.63811(8)$ & $0.33442(15)$ & $0.0481(5)$ \\
C9 & $0.4226(4)$ & $0.66836(8)$ & $0.25927(16)$ & $0.0481(5)$ \\
H9 & 0.4108 & 0.6615 & 0.1865 & $0.058^{*}$ \\
O2 & $1.1994(3)$ & $0.47252(7)$ & $0.42557(13)$ & $0.0667(4)$ \\
N2 & $0.0629(4)$ & $0.74082(8)$ & $0.21424(15)$ & $0.0570(4)$ \\
O1 & $1.2908(3)$ & $0.52526(7)$ & $0.26821(15)$ & $0.0720(5)$ \\
N1 & $0.9164(4)$ & $0.55519(7)$ & $0.36288(16)$ & $0.0534(4)$ \\
C10 & $0.2572(4)$ & $0.70859(8)$ & $0.29425(15)$ & $0.0471(5)$ \\
O3 & $0.8066(3)$ & $0.59550(7)$ & $0.19878(13)$ & $0.0695(5)$ \\
O4 & $-0.1159(4)$ & $0.76822(9)$ & $0.24399(15)$ & $0.0891(6)$ \\
O5 & $0.0900(4)$ & $0.73809(9)$ & $0.12131(13)$ & $0.0853(6)$ \\
C4 & $0.4935(5)$ & $0.38740(9)$ & $0.11605(18)$ & $0.0593(5)$ \\
C3 & $0.5450(5)$ & $0.38158(10)$ & $0.22531(18)$ & $0.0646(6)$ \\
H3 & 0.4508 & 0.3537 & 0.2563 & $0.077 *$
\end{tabular}




$\begin{array}{lllll}\text { C7 } & 0.7819(4) & 0.59512(9) & 0.29113(17) & 0.0518(5) \\ \text { C5 } & 0.6344(5) & 0.42936(10) & 0.07092(18) & 0.0680(6) \\ \text { H5 } & 0.6014 & 0.4341 & -0.0030 & 0.082^{*} \\ \text { C11 } & 0.2669(5) & 0.71991(9) & 0.40117(17) & 0.0583(5) \\ \text { H11 } & 0.1494 & 0.7467 & 0.4229 & 0.070^{*} \\ \text { C1 } & 0.8710(4) & 0.45716(8) & 0.24416(16) & 0.0490(5) \\ \text { C6 } & 0.8239(5) & 0.46431(10) & 0.13467(19) & 0.0630(6) \\ \text { H6 } & 0.9182 & 0.4923 & 0.1039 & 0.076^{*} \\ \text { C13 } & 0.6243(5) & 0.64965(9) & 0.44227(17) & 0.0602(6) \\ \text { H13 } & 0.7500 & 0.6298 & 0.4928 & 0.072^{*} \\ \text { C2 } & 0.7316(5) & 0.41580(10) & 0.29029(17) & 0.0588(5) \\ \text { H2 } & 0.7635 & 0.4111 & 0.3642 & 0.071^{*} \\ \text { C12 } & 0.4558(5) & 0.69042(10) & 0.47530(18) & 0.0669(6) \\ \text { H12 } & 0.4696 & 0.6981 & 0.5479 & 0.080^{*} \\ \text { C14 } & 0.2854(6) & 0.34997(12) & 0.0463(2) & 0.0845(8) \\ \text { H14A } & 0.1814 & 0.3292 & 0.0903 & 0.127^{*} \\ \text { H14B } & 0.3776 & 0.3232 & 0.0078 & 0.127^{*} \\ \text { H14C } & 0.1647 & 0.3736 & -0.0035 & 0.127^{*} \\ \text { HN1 } & 0.860(5) & 0.5495(10) & 0.417(2) & 0.062(8)^{*}\end{array}$

Atomic displacement parameters $\left(\AA^{2}\right)$

\begin{tabular}{lllllll}
\hline & $U^{11}$ & $U^{22}$ & $U^{33}$ & $U^{12}$ & $U^{13}$ & $U^{23}$ \\
\hline S1 & $0.0368(3)$ & $0.0518(3)$ & $0.0732(4)$ & $0.0021(2)$ & $0.0053(2)$ & $-0.0047(2)$ \\
C8 & $0.0445(11)$ & $0.0416(10)$ & $0.0566(11)$ & $-0.0023(8)$ & $0.0052(9)$ & $-0.0015(8)$ \\
C9 & $0.0469(11)$ & $0.0478(11)$ & $0.0488(10)$ & $-0.0051(9)$ & $0.0071(8)$ & $-0.0001(8)$ \\
O2 & $0.0538(9)$ & $0.0660(10)$ & $0.0726(10)$ & $0.0159(7)$ & $-0.0079(7)$ & $-0.0034(8)$ \\
N2 & $0.0582(11)$ & $0.0534(10)$ & $0.0563(11)$ & $0.0032(9)$ & $0.0024(8)$ & $0.0041(8)$ \\
O1 & $0.0428(8)$ & $0.0662(10)$ & $0.1106(13)$ & $-0.0065(7)$ & $0.0238(8)$ & $-0.0066(9)$ \\
N1 & $0.0444(10)$ & $0.0513(10)$ & $0.0627(11)$ & $0.0046(8)$ & $0.0053(8)$ & $-0.0017(8)$ \\
C10 & $0.0458(11)$ & $0.0423(10)$ & $0.0508(11)$ & $-0.0017(8)$ & $0.0027(8)$ & $0.0028(8)$ \\
O3 & $0.0759(11)$ & $0.0679(10)$ & $0.0705(11)$ & $0.0121(8)$ & $0.0292(8)$ & $0.0079(8)$ \\
O4 & $0.0871(13)$ & $0.0949(14)$ & $0.0821(12)$ & $0.0449(11)$ & $0.0077(10)$ & $0.0063(10)$ \\
O5 & $0.0957(14)$ & $0.1043(14)$ & $0.0529(10)$ & $0.0235(11)$ & $0.0063(9)$ & $0.0148(9)$ \\
C4 & $0.0611(13)$ & $0.0514(12)$ & $0.0619(13)$ & $0.0011(10)$ & $0.0025(10)$ & $-0.0020(10)$ \\
C3 & $0.0647(14)$ & $0.0599(13)$ & $0.0669(14)$ & $-0.0144(11)$ & $0.0066(11)$ & $0.0075(11)$ \\
C7 & $0.0448(11)$ & $0.0488(11)$ & $0.0612(13)$ & $-0.0026(9)$ & $0.0084(9)$ & $-0.0004(9)$ \\
C5 & $0.0866(17)$ & $0.0651(14)$ & $0.0516(12)$ & $-0.0038(12)$ & $0.0111(11)$ & $-0.0008(10)$ \\
C11 & $0.0665(14)$ & $0.0519(11)$ & $0.0551(12)$ & $0.0116(10)$ & $0.0082(10)$ & $-0.0036(9)$ \\
C1 & $0.0414(11)$ & $0.0471(10)$ & $0.0587(12)$ & $0.0034(8)$ & $0.0100(8)$ & $-0.0014(9)$ \\
C6 & $0.0686(15)$ & $0.0589(13)$ & $0.0647(13)$ & $-0.0080(11)$ & $0.0207(11)$ & $0.0028(10)$ \\
C13 & $0.0670(14)$ & $0.0536(12)$ & $0.0540(12)$ & $0.0093(10)$ & $-0.0041(10)$ & $0.0001(9)$ \\
C2 & $0.0591(13)$ & $0.0605(13)$ & $0.0546(12)$ & $-0.0081(10)$ & $0.0054(10)$ & $0.0051(10)$ \\
C12 & $0.0871(17)$ & $0.0623(14)$ & $0.0471(11)$ & $0.0166(12)$ & $0.0017(11)$ & $-0.0057(10)$ \\
C14 & $0.096(2)$ & $0.0701(16)$ & $0.0780(17)$ & $-0.0173(14)$ & $-0.0082(14)$ & $-0.0066(13)$ \\
& & & & & & \\
\hline & & & & & & \\
\hline
\end{tabular}


Geometric parameters $\left(\AA,{ }^{\circ}\right)$

\begin{tabular}{|c|c|c|c|}
\hline $\mathrm{S} 1-\mathrm{O} 1$ & $1.4156(17)$ & $\mathrm{C} 4-\mathrm{C} 14$ & $1.507(3)$ \\
\hline $\mathrm{S} 1-\mathrm{O} 2$ & $1.4305(16)$ & $\mathrm{C} 3-\mathrm{C} 2$ & $1.375(3)$ \\
\hline $\mathrm{S} 1-\mathrm{N} 1$ & $1.6580(18)$ & $\mathrm{C} 3-\mathrm{H} 3$ & 0.9300 \\
\hline $\mathrm{S} 1-\mathrm{C} 1$ & $1.747(2)$ & $\mathrm{C} 5-\mathrm{C} 6$ & $1.385(3)$ \\
\hline $\mathrm{C} 8-\mathrm{C} 13$ & $1.385(3)$ & $\mathrm{C} 5-\mathrm{H} 5$ & 0.9300 \\
\hline $\mathrm{C} 8-\mathrm{C} 9$ & $1.386(3)$ & $\mathrm{C} 11-\mathrm{C} 12$ & $1.382(3)$ \\
\hline $\mathrm{C} 8-\mathrm{C} 7$ & $1.495(3)$ & $\mathrm{C} 11-\mathrm{H} 11$ & 0.9300 \\
\hline $\mathrm{C} 9-\mathrm{C} 10$ & $1.373(3)$ & $\mathrm{C} 1-\mathrm{C} 6$ & $1.378(3)$ \\
\hline $\mathrm{C} 9-\mathrm{H} 9$ & 0.9300 & $\mathrm{C} 1-\mathrm{C} 2$ & $1.379(3)$ \\
\hline $\mathrm{N} 2-\mathrm{O} 4$ & $1.211(2)$ & $\mathrm{C} 6-\mathrm{H} 6$ & 0.9300 \\
\hline $\mathrm{N} 2-\mathrm{O} 5$ & $1.216(2)$ & $\mathrm{C} 13-\mathrm{C} 12$ & $1.382(3)$ \\
\hline $\mathrm{N} 2-\mathrm{C} 10$ & $1.470(3)$ & $\mathrm{C} 13-\mathrm{H} 13$ & 0.9300 \\
\hline $\mathrm{N} 1-\mathrm{C} 7$ & $1.383(3)$ & $\mathrm{C} 2-\mathrm{H} 2$ & 0.9300 \\
\hline $\mathrm{N} 1-\mathrm{HN} 1$ & $0.80(2)$ & $\mathrm{C} 12-\mathrm{H} 12$ & 0.9300 \\
\hline $\mathrm{C} 10-\mathrm{C} 11$ & $1.377(3)$ & $\mathrm{C} 14-\mathrm{H} 14 \mathrm{~A}$ & 0.9600 \\
\hline $\mathrm{O} 3-\mathrm{C} 7$ & $1.204(3)$ & $\mathrm{C} 14-\mathrm{H} 14 \mathrm{~B}$ & 0.9600 \\
\hline $\mathrm{C} 4-\mathrm{C} 3$ & $1.372(3)$ & $\mathrm{C} 14-\mathrm{H} 14 \mathrm{C}$ & 0.9600 \\
\hline $\mathrm{C} 4-\mathrm{C} 5$ & $1.387(3)$ & & \\
\hline $\mathrm{O} 1-\mathrm{S} 1-\mathrm{O} 2$ & $119.71(11)$ & $\mathrm{N} 1-\mathrm{C} 7-\mathrm{C} 8$ & $116.62(19)$ \\
\hline $\mathrm{O} 1-\mathrm{S} 1-\mathrm{N} 1$ & $108.51(10)$ & $\mathrm{C} 6-\mathrm{C} 5-\mathrm{C} 4$ & $120.8(2)$ \\
\hline $\mathrm{O} 2-\mathrm{S} 1-\mathrm{N} 1$ & $103.33(10)$ & $\mathrm{C} 6-\mathrm{C} 5-\mathrm{H} 5$ & 119.6 \\
\hline $\mathrm{O} 1-\mathrm{S} 1-\mathrm{C} 1$ & $109.63(10)$ & $\mathrm{C} 4-\mathrm{C} 5-\mathrm{H} 5$ & 119.6 \\
\hline $\mathrm{O} 2-\mathrm{S} 1-\mathrm{C} 1$ & $108.74(10)$ & $\mathrm{C} 10-\mathrm{C} 11-\mathrm{C} 12$ & $118.2(2)$ \\
\hline $\mathrm{N} 1-\mathrm{S} 1-\mathrm{C} 1$ & $105.96(9)$ & $\mathrm{C} 10-\mathrm{C} 11-\mathrm{H} 11$ & 120.9 \\
\hline $\mathrm{C} 13-\mathrm{C} 8-\mathrm{C} 9$ & $119.62(18)$ & $\mathrm{C} 12-\mathrm{C} 11-\mathrm{H} 11$ & 120.9 \\
\hline $\mathrm{C} 13-\mathrm{C} 8-\mathrm{C} 7$ & $124.23(18)$ & $\mathrm{C} 6-\mathrm{C} 1-\mathrm{C} 2$ & $120.7(2)$ \\
\hline $\mathrm{C} 9-\mathrm{C} 8-\mathrm{C} 7$ & $116.14(18)$ & $\mathrm{C} 6-\mathrm{C} 1-\mathrm{S} 1$ & $120.36(16)$ \\
\hline $\mathrm{C} 10-\mathrm{C} 9-\mathrm{C} 8$ & $118.78(18)$ & $\mathrm{C} 2-\mathrm{C} 1-\mathrm{S} 1$ & $118.94(16)$ \\
\hline $\mathrm{C} 10-\mathrm{C} 9-\mathrm{H} 9$ & 120.6 & $\mathrm{C} 1-\mathrm{C} 6-\mathrm{C} 5$ & $119.3(2)$ \\
\hline $\mathrm{C} 8-\mathrm{C} 9-\mathrm{H} 9$ & 120.6 & $\mathrm{C} 1-\mathrm{C} 6-\mathrm{H} 6$ & 120.4 \\
\hline $\mathrm{O} 4-\mathrm{N} 2-\mathrm{O} 5$ & $123.57(19)$ & $\mathrm{C} 5-\mathrm{C} 6-\mathrm{H} 6$ & 120.4 \\
\hline $\mathrm{O} 4-\mathrm{N} 2-\mathrm{C} 10$ & $118.53(19)$ & $\mathrm{C} 12-\mathrm{C} 13-\mathrm{C} 8$ & $120.42(19)$ \\
\hline $\mathrm{O} 5-\mathrm{N} 2-\mathrm{C} 10$ & $117.89(18)$ & $\mathrm{C} 12-\mathrm{C} 13-\mathrm{H} 13$ & 119.8 \\
\hline $\mathrm{C} 7-\mathrm{N} 1-\mathrm{S} 1$ & $123.00(17)$ & $\mathrm{C} 8-\mathrm{C} 13-\mathrm{H} 13$ & 119.8 \\
\hline $\mathrm{C} 7-\mathrm{N} 1-\mathrm{HN} 1$ & $118.4(18)$ & $\mathrm{C} 3-\mathrm{C} 2-\mathrm{C} 1$ & $119.0(2)$ \\
\hline $\mathrm{S} 1-\mathrm{N} 1-\mathrm{HN} 1$ & $114.5(17)$ & $\mathrm{C} 3-\mathrm{C} 2-\mathrm{H} 2$ & 120.5 \\
\hline $\mathrm{C} 9-\mathrm{C} 10-\mathrm{C} 11$ & $122.56(18)$ & $\mathrm{C} 1-\mathrm{C} 2-\mathrm{H} 2$ & 120.5 \\
\hline $\mathrm{C} 9-\mathrm{C} 10-\mathrm{N} 2$ & $118.58(17)$ & $\mathrm{C} 11-\mathrm{C} 12-\mathrm{C} 13$ & $120.4(2)$ \\
\hline $\mathrm{C} 11-\mathrm{C} 10-\mathrm{N} 2$ & $118.86(18)$ & $\mathrm{C} 11-\mathrm{C} 12-\mathrm{H} 12$ & 119.8 \\
\hline $\mathrm{C} 3-\mathrm{C} 4-\mathrm{C} 5$ & $118.4(2)$ & $\mathrm{C} 13-\mathrm{C} 12-\mathrm{H} 12$ & 119.8 \\
\hline $\mathrm{C} 3-\mathrm{C} 4-\mathrm{C} 14$ & $121.1(2)$ & $\mathrm{C} 4-\mathrm{C} 14-\mathrm{H} 14 \mathrm{~A}$ & 109.5 \\
\hline $\mathrm{C} 5-\mathrm{C} 4-\mathrm{C} 14$ & $120.5(2)$ & $\mathrm{C} 4-\mathrm{C} 14-\mathrm{H} 14 \mathrm{~B}$ & 109.5 \\
\hline $\mathrm{C} 4-\mathrm{C} 3-\mathrm{C} 2$ & $121.9(2)$ & $\mathrm{H} 14 \mathrm{~A}-\mathrm{C} 14-\mathrm{H} 14 \mathrm{~B}$ & 109.5 \\
\hline $\mathrm{C} 4-\mathrm{C} 3-\mathrm{H} 3$ & 119.0 & $\mathrm{C} 4-\mathrm{C} 14-\mathrm{H} 14 \mathrm{C}$ & 109.5 \\
\hline $\mathrm{C} 2-\mathrm{C} 3-\mathrm{H} 3$ & 119.0 & $\mathrm{H} 14 \mathrm{~A}-\mathrm{C} 14-\mathrm{H} 14 \mathrm{C}$ & 109.5 \\
\hline
\end{tabular}




$\begin{array}{llll}\mathrm{O} 3-\mathrm{C} 7-\mathrm{N} 1 & 121.6(2) & \mathrm{H} 14 \mathrm{~B}-\mathrm{C} 14-\mathrm{H} 14 \mathrm{C} & 109.5 \\ \mathrm{O} 3-\mathrm{C} 7-\mathrm{C} 8 & 121.82(19) & & \\ \mathrm{C} 13-\mathrm{C} 8-\mathrm{C} 9-\mathrm{C} 10 & -1.0(3) & \mathrm{C} 14-\mathrm{C} 4-\mathrm{C} 5-\mathrm{C} 6 & 179.3(2) \\ \mathrm{C} 7-\mathrm{C} 8-\mathrm{C} 9-\mathrm{C} 10 & -179.78(17) & \mathrm{C} 9-\mathrm{C} 10-\mathrm{C} 11-\mathrm{C} 12 & 1.7(3) \\ \mathrm{O} 1-\mathrm{S} 1-\mathrm{N} 1-\mathrm{C} 7 & 51.79(19) & \mathrm{N} 2-\mathrm{C} 10-\mathrm{C} 11-\mathrm{C} 12 & -178.2(2) \\ \mathrm{O} 2-\mathrm{S} 1-\mathrm{N} 1-\mathrm{C} 7 & 179.85(17) & \mathrm{O} 1-\mathrm{S} 1-\mathrm{C} 1-\mathrm{C} 6 & -23.4(2) \\ \mathrm{C} 1-\mathrm{S} 1-\mathrm{N} 1-\mathrm{C} 7 & -65.87(19) & \mathrm{O} 2-\mathrm{S} 1-\mathrm{C} 1-\mathrm{C} 6 & -155.95(17) \\ \mathrm{C} 8-\mathrm{C} 9-\mathrm{C} 10-\mathrm{C} 11 & -0.3(3) & \mathrm{N} 1-\mathrm{S} 1-\mathrm{C} 1-\mathrm{C} 6 & 93.55(19) \\ \mathrm{C} 8-\mathrm{C} 9-\mathrm{C} 10-\mathrm{N} 2 & 179.61(17) & \mathrm{O} 1-\mathrm{S} 1-\mathrm{C} 1-\mathrm{C} 2 & 158.45(17) \\ \mathrm{O} 4-\mathrm{N} 2-\mathrm{C} 10-\mathrm{C} 9 & 166.6(2) & \mathrm{O} 2-\mathrm{S} 1-\mathrm{C} 1-\mathrm{C} 2 & -84.63(19) \\ \mathrm{O} 5-\mathrm{N} 2-\mathrm{C} 10-\mathrm{C} 9 & -12.9(3) & \mathrm{N} 1-\mathrm{S} 1-\mathrm{C} 1-\mathrm{C} 2 & -0.1(3) \\ \mathrm{O} 4-\mathrm{N} 2-\mathrm{C} 10-\mathrm{C} 11 & -13.5(3) & \mathrm{C} 2-\mathrm{C} 1-\mathrm{C} 6-\mathrm{C} 5 & -178.20(18) \\ \mathrm{O} 5-\mathrm{N} 2-\mathrm{C} 10-\mathrm{C} 11 & 167.0(2) & \mathrm{S} 1-\mathrm{C} 1-\mathrm{C} 6-\mathrm{C} 5 & -0.2(4) \\ \mathrm{C} 5-\mathrm{C} 4-\mathrm{C} 3-\mathrm{C} 2 & -0.3(4) & \mathrm{C} 4-\mathrm{C} 5-\mathrm{C} 6-\mathrm{C} 1 & 1.0(3) \\ \mathrm{C} 14-\mathrm{C} 4-\mathrm{C} 3-\mathrm{C} 2 & -179.2(2) & \mathrm{C} 9-\mathrm{C} 8-\mathrm{C} 13-\mathrm{C} 12 & 0.0(4) \\ \mathrm{S} 1-\mathrm{N} 1-\mathrm{C} 7-\mathrm{O} 3 & -4.9(3) & \mathrm{C} 7-\mathrm{C} 8-\mathrm{C} 13-\mathrm{C} 12 & 0.2(3) \\ \mathrm{S} 1-\mathrm{N} 1-\mathrm{C} 7-\mathrm{C} 8 & 175.83(14) & \mathrm{C} 4-\mathrm{C} 3-\mathrm{C} 2-\mathrm{C} 1 & 178.32(18) \\ \mathrm{C} 13-\mathrm{C} 8-\mathrm{C} 7-\mathrm{O} 3 & -162.5(2) & \mathrm{C} 6-\mathrm{C} 1-\mathrm{C} 2-\mathrm{C} 3 & -1.7(4) \\ \mathrm{C} 9-\mathrm{C} 8-\mathrm{C} 7-\mathrm{O} 3 & 16.1(3) & \mathrm{S} 1-\mathrm{C} 1-\mathrm{C} 2-\mathrm{C} 3 & 0.4(4) \\ \mathrm{C} 13-\mathrm{C} 8-\mathrm{C} 7-\mathrm{N} 1 & 16.8(3) & \mathrm{C} 10-\mathrm{C} 11-\mathrm{C} 12-\mathrm{C} 13 & \\ \mathrm{C} 9-\mathrm{C} 8-\mathrm{C} 7-\mathrm{N} 1 & -164.58(18) & \mathrm{C} 8-\mathrm{C} 13-\mathrm{C} 12-\mathrm{C} 11 & \\ \mathrm{C} 3-\mathrm{C} 4-\mathrm{C} 5-\mathrm{C} 6 & 0.4(4) & & \\ & & & \end{array}$

Hydrogen-bond geometry $\left(\AA,{ }^{\circ}\right)$

\begin{tabular}{lllll}
\hline$D-\mathrm{H} \cdots A$ & $D-\mathrm{H}$ & $\mathrm{H} \cdots A$ & $D \cdots A$ & $D-\mathrm{H}^{\cdots} A$ \\
\hline $\mathrm{N} 1-\mathrm{H} N 1 \cdots \mathrm{O} 2^{\mathrm{i}}$ & $0.80(3)$ & $2.14(3)$ & $2.927(3)$ & 167 \\
$\mathrm{C} 13-\mathrm{H} 13 \cdots \mathrm{O} 2^{\mathrm{i}}$ & 0.93 & 2.59 & $3.333(3)$ & 137 \\
$\mathrm{C} 3-\mathrm{H} 3 \cdots \mathrm{O} 4^{\mathrm{ii}}$ & 0.93 & 2.58 & $3.459(3)$ & 155 \\
\hline
\end{tabular}

Symmetry codes: (i) $-x+2,-y+1,-z+1$; (ii) $-x, y-1 / 2,-z+1 / 2$. 